\title{
Effect of pathological fracture on limb salvage surgery with preservation of the epiphysis in children with osteosarcoma of the distal femur: Two case reports
}

\author{
KAI ZHENG, XIUCHUN YU, ZHENGQI CHANG, SONGFENG XU and MING XU
}

Department of Orthopedics, The General Hospital of Jinan Military Commanding Region, Jinan, Shandong 250031, P.R. China

Received July 15, 2015; Accepted February 3, 2016

DOI: $10.3892 / \mathrm{mco} .2016 .777$

\begin{abstract}
The outcome of limb salvage treatment for femoral osteosarcoma with pathological fractures in children is currently unknown. The aim of the present study was to present two cases of patients who received limb salvage surgery with preservation of the epiphysis at the Department of Orthopedics of The General Hospital of Jinan Military Commanding Region (Shandong, China). Between January, 2007 and January, 2013, two pediatric patients were admitted to our hospital with pathological fractures. One of the patients was a girl, aged 11 years, with confirmed osteosarcoma of the right distal femur; the other patient was a boy, aged 9 years, with osteosarcoma of the left distal femur. After receiving two cycles of neoadjuvant chemotherapy following tumor biopsy, the patients received limb salvage surgery with epiphyseal preservation, with wide resection of the tumor and biological reconstruction by allogeneic bone and fibular autograft, followed by 10 cycles of adjuvant chemotherapy. With a mean follow-up of 64 months, there were no postoperative complications, local recurrence or metastasis. The limb function recovered well, although limb shortening was observed. The female patient underwent a second fixation and limb lengthening after epiphyseal closure. Therefore, with effective neoadjuvant chemotherapy, limb salvage surgery with epiphyseal preservation is not contraindicated for pediatric patients with pathological fractures from femoral osteosarcoma. Biological reconstruction by allogeneic bone and vascularized fibular autograft following wide tumor resection is a viable option for such patients, with a good postoperative functional outcome.
\end{abstract}

\section{Introduction}

Osteosarcoma is the most common solid malignancy of the bone, with an incidence of 2-3 per 106,000 individuals, but

Correspondence to: Dr Xiuchun Yu, Department of Orthopedics, The General Hospital of Jinan Military Commanding Region, 25 Shifan Street, Jinan, Shandong 250031, P.R. China

E-mail: yxch48@vip.sina.com

Key words: osteosarcoma, pathological fracture, limb salvage, epiphyseal preservation, biological reconstruction
8-11 per 106,000 individuals aged $15-19$ years (1). Metaphyseal osteosarcoma of the femur with a pathological fracture is common among children (2); it occurs most frequently around the knee, whereas $\sim 75 \%$ of the cases are located near the epiphyseal plate (3), with an incidence of complicating pathological fractures of $\sim 5 \%$ (4). However, metaphyseal osteosarcoma in children has been widely considered as a contraindication for limb salvage surgery (2). First, pathological fracture results in loss of the surgical border of the tumor; thus, radical or wide resection cannot be performed $(5,6)$. Second, reconstruction with prosthetic replacement is not suitable for children who have yet to reach skeletal maturity. Third, due to the complexity of biological reconstruction and limb shortening in the future, certain surgeons consider rotationplasty to be a suitable option for children following tumor resection around the knee (7). In this study, we present two cases of pediatric patients who received limb salvage treatment with preservation of the epiphysis, including wide resection of the tumor and biological reconstruction by allogeneic bone and fibular autograft. We recommend this method for children with distal femoral osteosarcoma complicated by pathological fracture.

\section{Case reports}

Case 1. The first patient was a girl aged 11 years who experienced pain in the lower section of the left thigh for 5 months. X-ray examination at another hospital in March, 2007 revealed bone destruction (Fig. 1A) and anti-inflammatory treatment was administered, but without subsidence of the pain. The patient was admitted to the Department of Orthopedics of The General Hospital of Jinan Military Commanding Region (Shandong, China) in August, 2007, as the pain had significantly worsened and the tumor grew rapidly. On physical examination, edema and deformation were observed. X-ray examination revealed bone destruction and a fracture of the left distal femur (Fig. 1B). The left leg was immobilized with plaster external fixation and the pathological examination following needle aspiration biopsy confirmed the diagnosis of osteosarcoma. Preoperative chemotherapy, containing cisplatin (DDP) $100 \mathrm{mg} / \mathrm{m}^{2}$, doxorubicin (ADM) $20 \mathrm{mg} / \mathrm{m}^{2}$ and ifosfamide (IFO) $2 \mathrm{~g} / \mathrm{m}^{2}$, was immediately administered. After two cycles, the symptoms gradually improved. Wide resection including the epiphyseal plate was performed to ensure safe boundaries, followed by biological reconstruction by allogeneic bone and 
avascular fibular autograft for the bone defect. The follow-up $\mathrm{X}$-ray at 6 months post-operation revealed an external callus in the proximal bone stump, but not in the distal (Fig. 1C). The follow-up X-ray at 18 months post-operation revealed successful bone healing in the proximal end, but allograft bone resorption was obvious in the distal end (Fig. 1D). A second surgery was undertaken, which included autogenous bone graft and internal fixation. The patient resumed normal activity following postoperative rehabilitation. The patient revisited our hospital in July, 2012, 5 years after tumor treatment, due to limb shortening (Fig. 1E). In order to improve limb function, limb lengthening in the proximal femur with external fixation was undertaken following X-ray confirmation of epiphyseal closure. The follow-up X-ray showed an obvious limb lengthening and the external fixation was removed after that (Fig. 1F-G). On further follow-up, the patient had good limb function and led a normal life.

Case 2. The second patient was a boy aged 9 years, who was admitted for dysfunction of the right knee in October, 2012. The patient had experienced pain in the lower part of the right thigh for 2 months, and a mass was detected 1 week prior to admission. The patient also complained of night pain. On physical examination, there was a deformation of the right femur and elevation of local skin temperature. Subcutaneous veins were not apparent and a mass sized $6 \times 6 \mathrm{~cm}$ was detected on palpation. Radiography revealed osteolytic destruction, discontinuity of the cortex, intercalation fracture with angular deformity and soft tissue edema of the right distal femur. There was no obvious periosteal reaction or callus formation (Fig. 2A). The tumor was confirmed to be osteosarcoma following needle aspiration biopsy. Preoperative chemotherapy with DDP, ADM and IFO was immediately administered. After two cycles, the symptoms gradually improved. An X-ray revealed osteosclerosis and reactive periosteal bone formation. There was no soft tissue involvement (Fig. 2B). Magnetic resonance imaging (MRI) revealed that the osteoepiphysis proximal to the metaphyseal region was not interrupted by the tumor. Moreover, the body of the tumor had already shrunk and the edema had decreased (Fig. 2C). The patient underwent surgery following preoperative chemotherapy. A homolateral vascularized fibular autograft was prepared, as reported previously (9). The lower margin of the tumor was resected, including the epiphyseal plate, and the other margins were removed by wide resection (Fig. 2D). After the limb length was restored, an allogeneic bone of appropriate size was transplanted with the intercalated vascularized fibular autograft (Fig. 2E). The region was fixed with a locking plate. The peroneal artery and vein were anastomosed to the lateral superior genicular artery and vein, respectively. The blood flow in the region of the distal fibula was immediately restored. Postoperatively, no complications occurred. The postoperative histological findings confirmed a negative surgical margin. At 2 weeks post-operation, chemotherapy, which was identical to the preoperative regimen, was administered for 10 cycles. Movement of the knee joint was allowed 3 months postoperatively and full weight-bearing mobility was initiated at 6 months postoperatively. On further follow-up at 8 months post-operation, there was no limb length discrepancy or knee deformity, and the range of motion in the right knee was 0-100 degrees. Radiography revealed that the transplanted bone had healed and there was no evidence of metastasis or local recurrence (Fig. 2F).

\section{Discussion}

Osteosarcoma is the most common primary malignant bone tumor in children and young adults $(4,8)$. Approximately $5-20 \%$ of the patients suffering from osteosarcoma develop pathological fractures during the course of preoperative treatment $(4,9)$. The prognostic significance of a pathological fracture in patients with an osteosarcoma is widely debated. It is accepted that malignant bone tumors with pathological fractures often lead to compartment structure damage and tumor cell spread, which makes surgeons consider amputation as the most suitable treatment in order to avoid the dissemination of tumor cells. However, Abudu et al consider amputation to be a radical resection method for a local tumor, but cannot prolong survival time (4). With the gradual development of neoadjuvant chemotherapy, several researchers have reported that it may be feasible to salvage the limb with pathological fractures during osteosarcoma therapy $(5,10-12)$. In this study, two pediatric patients received neoadjuvant chemotherapy, which made the tumor boundaries clearer and helped achieve a wide resection. Limb salvage surgery with epiphyseal preservation was successful for the two patients, and comparable with other osteosarcoma patients without pathological fractures.

There are several advantages of epiphyseal preservation in the removal of malignant bone tumors in children. However, it remains unclear how to achieve safe resection in osteosarcoma with pathological fractures. Aponte-Tinao et al (13) reported that a margin of $\geq 1 \mathrm{~cm}$ of normal bone from the tumor on MRI imaging was a safe resection margin for osteosarcoma. Epiphyseal preservation may not be suitable for some patients. When tumor cells are found in the physeal margin, trans-epiphyseal resection or knee arthroplasty is considered to be the appropriate surgical treatment, rather than physeal preservation. Prosthesis replacement in children presents with a number of difficulties, including non-standard prosthesis size, prosthesis replaced several times and other surgical complications, although the new extendable prosthesis is currently recommended. A previous study indicated that prosthetic survival rates for large-segment replacements in high-grade bone sarcoma were $83 \%$ at 5 years and $67 \%$ at 10 years (14). There are several methods for biological reconstruction following excision of peri-articular osteosarcomas around the knee joint, including distraction osteogenesis, intercalary allograft, vascularized fibular graft, frozen autograft, or osteoarticular allograft. Manfrini et al described a case using a fibular graft including the proximal joint cartilage and the growth plate for reconstruction of the proximal femur and hip joint in an osteosarcoma patient (15). In this study, a vascularized fibular graft and allogeneic bone of similar size were used for repairing the bone defect for biological reconstruction. The joint function was preserved and the patients returned to normal activity after bone healing. Even if shortening does occur, simple lengthening may solve the problem. The female patient in this study underwent limb lengthening in the proximal femur with external fixation after the X-ray confirmed epiphyseal closure. 


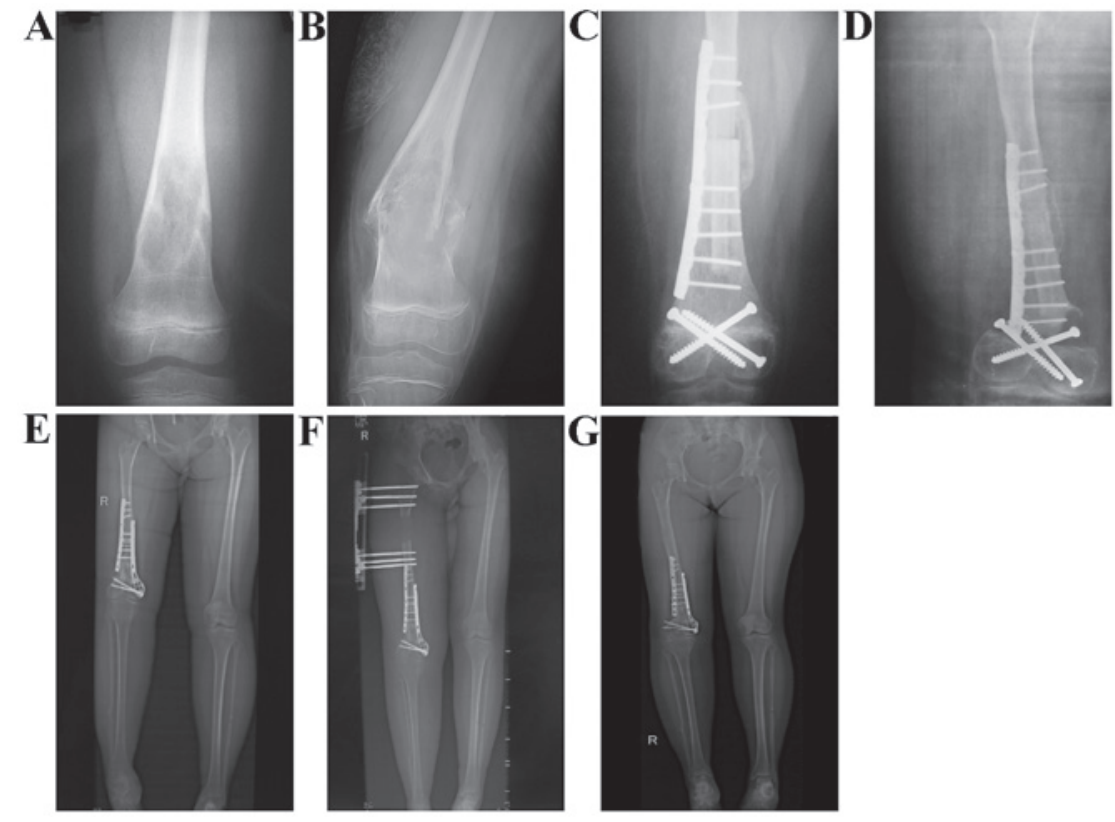

Figure 1. (A) X-ray image obtained in another hospital and (B) X-ray 5 months later. (C) The follow-up X-ray at 6 months post-operation revealed external callus formation in the proximal bone stump, but not in distal. (D) The follow-up X-ray at 18 months post-operation revealed successful bone healing in the proximal stump but allograft bone resorption in the distal stump. (E) The patient visited our hospital due to limb shortening 5 years after tumor treatment. (F) Limb lengthening in the proximal femur with external fixation was undertaken after the X-ray confirmed epiphyseal closure. (G) The follow-up X-ray revealed obvious limb lengthening, after which time the external fixation was removed.

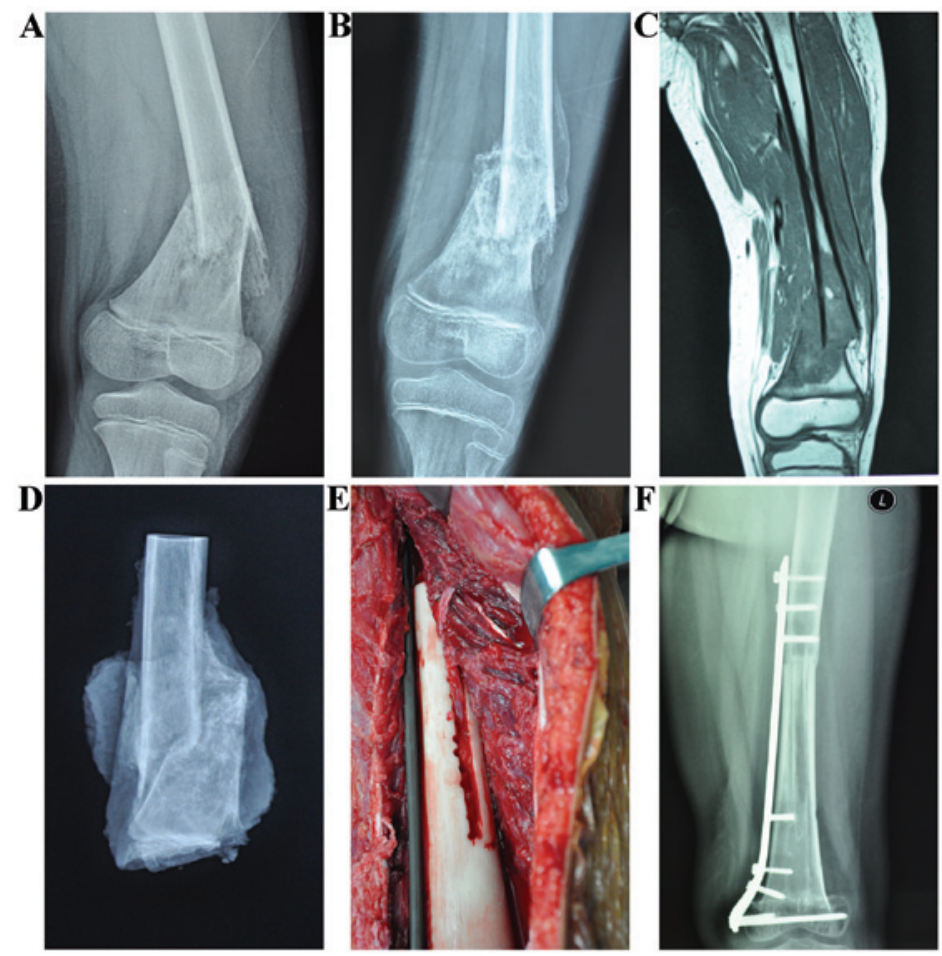

Figure 2. X-ray imaging (A) before and (B) after chemotherapy. (C) Magnetic resonance imaging after chemotherapy. (D) The tumor was removed by extensive resection. (E) Allogeneic bone of appropriate size was transplanted with an intercalated vascularized fibular autograft. (F) X-ray of the grafts at 8 months post-operation.

In children, the intensity of biological reconstruction following tumor resection is crucial. Aponte-Tinao et al (13) analyzed 35 osteosarcoma patients with epiphyseal preservation and allograft reconstruction, with a minimum follow-up of 5 years, and reported 11 fractures (31\%) in their series. Weitao et al (16) analyzed 15 patients who underwent epiphysis-preserving procedures for the treatment of malignant bone tumors of the lower limb, with a minimum follow-up of 2 years, who had reconstruction with an intercalary allograft; they reported 13 patients with delayed union $(87 \%)$. As is well known, bone healing between allogeneic bone and preserved bone is slow; the vascularized fibular graft inside 
the allogeneic bone accelerates bone healing and the fibular graft combined with allogeneic bone ensure sufficient bone strength. In this study, allograft bone resorption occurred distally in case 1 , but not in case 2 , the possible reasons being that vascularized fibular graft is superior to avascular fibular graft, and rigid internal fixation with a plate is preferred to fixation with screws.

Such cases are not common. Three pediatric patients with femoral osteosarcoma with pathological fractures were treated in our hospital between January, 2005 and January, 2015. One patient underwent knee replacement following an unfavorable outcome with chemotherapy, and eventually succumbed to lung metastasis 10 months after the operation. In the two patients described herein, the pathological fracture did not lead to tumor cell dissemination into the knee joint, which made joint preservation possible. Another important factor that made limb salvage surgery possible, was that the osteosarcoma in our two cases responded well to chemotherapy. The advantages of this reconstruction are preservation of a normal joint in children, the possibility of avoiding the joint complications seen with osteoarticular grafts (eventual need for conversion to total knee arthroplasty, joint instability) and endoprostheses (loosening, revisions). However, this biological reconstruction must be performed on a background of effective chemotherapy, a safe tumor resection margin, and a surgical team experienced with microsurgical techniques. In conclusion, limb salvage surgery with epiphyseal preservation is not contraindicated for pediatric patients with pathological fractures from femoral osteosarcoma.

\section{References}

1. Stiller CA, Bielack SS, Jundt G and Steliarova-Foucher E: Bone tumours in European children and adolescents, 1978-1997. Report from the Automated Childhood Cancer Information System project. Eur J Cancer 42: 2124-2135, 2006.

2. San-Julian M, Aquerreta JD, Benito A and Cañadell J: Indications for epiphyseal preservation in metaphyseal malignant bone tumors of children: Relationship between image methods and histological findings. J Pediatr Orthop 19: 543-548, 1999.
3. Cara JA and Canadell J: Limb salvage for malignant bone tumors in young children. J Pediatr Orthop 14: 112-118, 1994.

4. Abudu A, Sferopoulos NK, Tillman RM, Carter SR and Grimer RJ: The surgical treatment and outcome of pathological fractures in localised osteosarcoma. J Bone Joint Surg Br 78: 694-698, 1996.

5. Salunke AA, Chen Y, Tan JH, Chen X, Khin LW and Puhaindran ME: Does a pathological fracture affect the prognosis in patients with osteosarcoma of the extremities? A systematic review and meta-analysis. Bone Joint J 96-B: 1396-1403, 2014.

6. Lee RK, Chu WC, Leung JH, Cheng FW and Li CK: Pathological fracture as the presenting feature in pediatric osteosarcoma. Pediatr Blood Cancer 60: 1118-1121, 2013.

7. Merkel KD, Gebhardt M and Springfield DS: Rotationplasty as a reconstructive operation after tumor resection. Clin Orthop Relat Res 270: 231-236, 1991.

8. Glass AG and Fraumeni JF Jr: Epidemiology of bone cancer in children. J Natl Cancer Inst 44: 187-199, 1970.

9. Jaffe N, Spears R, Eftekhari F, Robertson R, Cangir A, Takaue Y, Carrasco H, Wallace S, Ayala A and Raymond K: Pathologic fracture in osteosarcoma. Impact of chemotherapy on primary tumor and survival. Cancer 59: 701-709, 1987.

10. Xie L, Guo W, Li Y, Ji T and Sun X: Pathologic fracture does not influence local recurrence and survival in high-grade extremity osteosarcoma with adequate surgical margins. J Surg Oncol 106: 820-825, 2012.

11. Cui Q, Li DF, Liu C, Guo J, Liu SB, Liu YS, Zhai JM and Zeng Y: Two case-reports of the limb salvage treatment of osteosarcoma consolidated with obvious pathological fractures. Pathol Oncol Res 17: 973-979, 2011.

12. Zuo D, Zheng L, Sun W, Hua Y and Cai Z: Pathologic fracture does not influence prognosis in stage IIB osteosarcoma: A case-control study. World J Surg Oncol 11: 148, 2013.

13. Aponte-Tinao L, Ayerza MA, Muscolo DL and Farfalli GL: Survival, recurrence and function after epiphyseal preservation and allograft reconstruction in osteosarcoma of the knee. Clin Orthop Relat Res 473: 1789-1796, 2015.

14. Malawer MM and Chou LB: Prosthetic survival and clinical results with use of large-segment replacements in the treatment of high-grade bone sarcomas. J Bone Joint Surg Am 77: 1154-1165, 1995.

15. Manfrini M, Innocenti M, Ceruso $M$ and Mercuri M: Original biological reconstruction of the hip in a 4-year-old girl. Lancet 361: 140-142, 2003.

16. Weitao Y, Qiqing C, Songtao G and Jiaqiang W: Epiphysis preserving operations for the treatment of lower limb malignant bone tumors. Eur J Surg Oncol 38: 1165-1170, 2012. 